\title{
MECHANICAL AND ELECTRICAL PROPERTIES OF TERNARY Ag-Bi-Ga SYSTEM AT $250^{\circ} \mathrm{C}$
}

\author{
Duško Minić ${ }^{1}$, Dragan Manasijević2 ${ }^{2}$,Yong Dư ${ }^{3}$, Pavel Brož́, \\ Vladan Ćosović, Aleksandar Đorđević ${ }^{1}$ and Milena Premović ${ }^{1,3, *}$ \\ ${ }^{11}$ University of Priština, Faculty of Technical Science, Kneza Milosa 7, \\ Kos. Mitrovica, Serbia \\ ${ }^{2}$ University of Belgrade, Technical Faculty in Bor, VJ 12, 19210 Bor, Serbia \\ ${ }^{3}$ State Key Laboratory of Powder Metallurgy, Central South University, \\ Changsha, PR China \\ ${ }^{4}$ Masaryk University, Faculty of Science, Department of Chemistry, Brno, \\ Czech Republic \\ ${ }^{5}$ University of Belgrade, Institute of Chemistry, Technology and Metallurgy, \\ Belgrade, Serbia
}

Received 02.08.2017

Accepted 01.09.2017

\begin{abstract}
This paper presents a comparative review of the experimental and thermodynamic assessment of a ternary Ag-Bi-Ga system. An isothermal section at 250 ${ }^{\circ} \mathrm{C}$ was calculated using optimized thermodynamic data for the constitutive binaries. Microstructures and phase compositions of studied alloys were analyzed by scanning electron microscopy in combination with energy dispersive spectrometry and X-ray powder diffraction technique. The obtained experimental results were found to support the predicted phase equilibria rather well. The hardness of alloys from three vertical sections (Bi-AgGa, $\mathrm{Ag}-\mathrm{BiGa}$, and $\mathrm{Ga}-\mathrm{AgBi}$ ) was determined using Brinell hardness test while the hardness of the individual identified phases was determined using Vickers microhardness test. Additional electrical conductivity measurements were carried out on the same alloy samples. Based on the experimentally obtained results iso-lines of Brinell hardness and electrical conductivity for the entire compositional range were calculated.
\end{abstract}

Keywords: materials testing; isothermal section at $250{ }^{\circ} \mathrm{C}$; microstructure; hardness; electrical conductivity.

\footnotetext{
*Corresponding author: Milena Premović,milena.premovic@gmail.com
} 


\section{Introduction}

The ternary Ag-Bi-Ga system was thoroughly investigated in our previous study (Minic et al.) [1]. The results of experimental investigations of the liquidus surface; three vertical sections and the isothermal section at $200{ }^{\circ} \mathrm{C}$ are presented in the paper [1]. However; in the current study, the focus is on the experimental investigation of mechanical and electrical properties of alloys of the ternary Ag-Bi-Ga system. Besides mentioned properties; the paper also presents results of the SEM-EDS and XRD analysis for alloys from the isothermal section at $250^{\circ} \mathrm{C}$. The selected temperature (250 ${ }^{\circ} \mathrm{C}$ ) is rather interesting for investigation since the largest number of different phases is in equilibrium at this temperature and also because at temperatures higher than $2500^{\circ} \mathrm{C}$ liquidus phases are dominant (melting point of included elements are Ag- $961.78{ }^{\circ} \mathrm{C}$; Ga-29.76 ${ }^{\circ} \mathrm{C}$ and $\left.\mathrm{Bi}-271.3{ }^{\circ} \mathrm{C}[2]\right)$.

The concept of this study and the other similar studies [3 - 5] provides the possibility to follow up how the properties of a base element are changing with the addition of other elements. The significance of the selected ternary system itself reflects in a fact that; as widely known; owing to its outstanding heat and electrical conductivity the silver is practically irreplaceable for various special applications. Also, it is well known that the Ag-Bi $[6,7]$ or Ag-Ga $[8,9]$ binaries are extensively used for welding; soldering; in electronics and in the chemical industry.

\section{Experimental procedure}

All investigated samples were prepared from Ag; Bi; and Ga shots of 99.99 at. \% purity in an induction furnace under high-purity argon atmosphere. In general; the average loss of mass during melting of samples is about 2 mass \%. Before annealing; the samples were sealed in evacuated quartz tubes. After that; the prepared samples were heated to the temperature that is $50{ }^{\circ} \mathrm{C}$ higher than the melting point of $\mathrm{Ag}$ and then cooled to $250^{\circ} \mathrm{C}$ at a cooling rate of $5{ }^{\circ} \mathrm{C} \mathrm{min}-1$. The samples for all investigation were annealed at $250{ }^{\circ} \mathrm{C}$ for 2 months and subsequently quenched into an ice/water mixture in order to preserve the desired equilibrium.

The microstructure of the prepared alloy samples was studied using TESCAN VEGA3 scanning electron microscope in combination with energy dispersive spectroscopy (EDS) (Oxford Instruments X-act). The samples were prepared by the standard metallographic procedure without etching. The polished alloy samples were initially inspected using EDS elemental mapping in order to check compositional homogeneity and possible segregation. Overall compositions of the samples, as well as compositions of coexisting phases, were subsequently determined using EDS point and area analysis.

XRD patterns of the studied samples were obtained using D2 PHASER powder diffractometer with a dynamic scintillation detector and ceramic $\mathrm{X}$-ray $\mathrm{Cu}$ tube (KFLCu-2K) in a 5 to $75^{\circ} 2 \theta$ range with 0.02 deg step size. The recorded patterns were subsequently analyzed using Topas 4.2 software and ICDD databases PDF2 (2013).

Brinell hardness tester INNOVATEST; model Nexus 3001 was used for testing of the samples while the microhardness of the individual phases was determined using a Vickers microhardness tester Sinowon; model Vexus ZHV-1000V. The electrical conductivity of the studied alloys was measured using Foerster SIGMATEST 2.069 eddy current instrument. 


\section{Literature data}

The isothermal section at $250{ }^{\circ} \mathrm{C}$ of the ternary $\mathrm{Ag}-\mathrm{Bi}-\mathrm{Ga}$ system has been thermodynamically predicted using optimized thermodynamic parameters for the constitutive binaries from literature $\left[\begin{array}{ll}10 & -12\end{array}\right]$. The optimized thermodynamic parameters for the binary Ag-Ga system were taken from Gierlotka et al. [10]. Likewise; the parameters for the Ag-Bi system were taken from Zoro et al. [11] and for the Bi-Ga binary system from Girard [12]. Thermodynamic calculations were carried out by using PANDAT software package [13].

A list of phases from the binary subsystems considered for thermodynamic binary-based prediction is given in Table 1 together with their corresponding Pearson symbols.

Table 1. Phases considered and their crystal structures [14, 15].

\begin{tabular}{ccccc}
\hline $\begin{array}{c}\text { Thermodynamic } \\
\text { database name }\end{array}$ & Phase & $\begin{array}{c}\text { Strukturbericht } \\
\text { designation }\end{array}$ & $\begin{array}{c}\text { Pearson } \\
\text { symbol }\end{array}$ & $\begin{array}{c}\text { Space } \\
\text { group }\end{array}$ \\
\hline Liquid & Liquid & & & \\
Fcc_A1 & $(\mathrm{Ag})$ & $\mathrm{A} 1$ & $c F 4$ & $F m \overline{3} m$ \\
Rhombo_A7 & $(\mathrm{Bi})$ & $\mathrm{A} 7$ & $h R 2$ & $R \overline{3} m$ \\
Ortho_Ga & $(\mathrm{Ga})$ & $\mathrm{A} 11$ & $o C 8$ & $C m c a$ \\
Hcp_A3 & $\zeta-\mathrm{Ag}_{2} \mathrm{Ga}$ & $\mathrm{A} 3$ & $h P 2$ & $P 6_{3} / m m c$ \\
$\mathrm{Hcp}$ _ord & $\zeta^{\prime}-\mathrm{Ag}_{2} \mathrm{Ga}$ & $\mathrm{C} 22$ & $h P 9$ & $P \overline{6} 2 m$ \\
$\mathrm{Ag}_{3} \mathrm{Ga}_{2}$ & $\mathrm{Ag}_{3} \mathrm{Ga}_{2}$ & - & - & $P m m m$ \\
\hline
\end{tabular}

\section{Results and discussion}

Fig. 1 presents calculated isothermal section at $250{ }^{\circ} \mathrm{C}$ of the Ag-Bi-Ga ternary system. The alloy samples selected for microstructural analysis by SEM-EDS and XRD techniques are marked on Fig. 1 by corresponding symbols. Dashed orange lines are shown on Fig. 1 mark three vertical sections along which lay the compositions of the alloy samples used for hardness and electrical conductivity measurements.

From fourteen regions predicted in the isothermal section $250{ }^{\circ} \mathrm{C}$; eight of them were investigated using SEM-EDS and XRD experimental techniques. The obtained EDS results are given in Table 2 in comparison with the predicted phase compositions. 


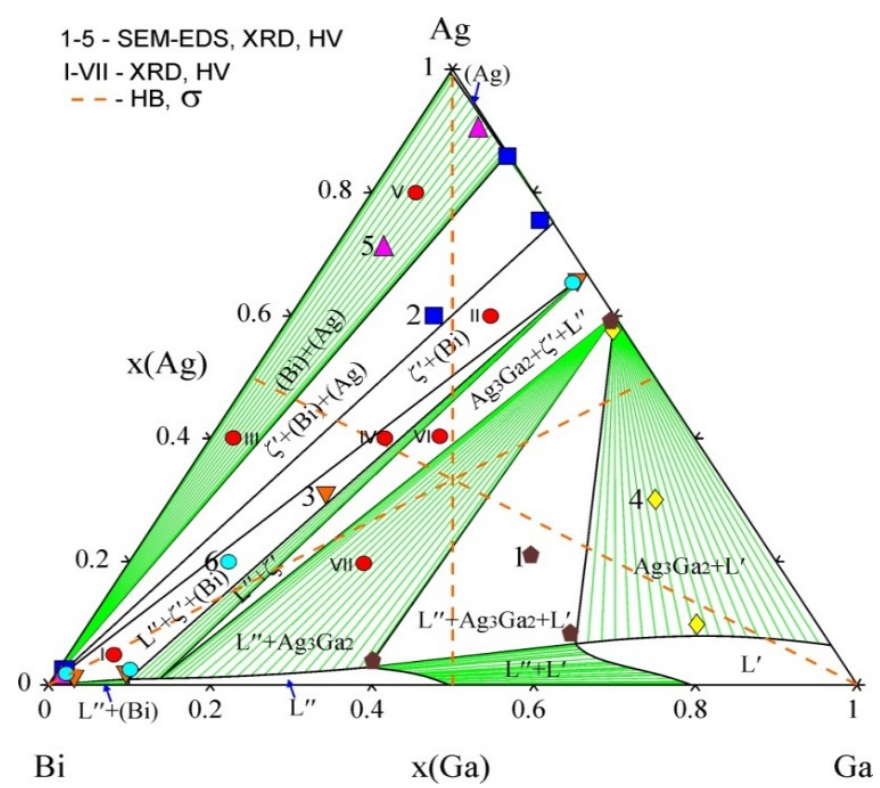

Fig. 1. Isothermal sections of the ternary $\mathrm{Ag}$-Bi-Ga systems at $250^{\circ} \mathrm{C}$ with marked overall compositions of investigated samples.

Table 2. Calculated and experimentally determined phase compositions in the ternary Ag-Bi-Ga system at $250^{\circ} \mathrm{C}$.

\begin{tabular}{|c|c|c|c|c|c|c|c|c|c|}
\hline \multirow{3}{*}{ S. } & \multirow{3}{*}{$\begin{array}{l}\text { Overall } \\
\text { composition } \\
\text { (at.\%) }\end{array}$} & \multirow{3}{*}{$\begin{array}{l}\text { Theor. } \\
\text { predicted } \\
\text { phases }\end{array}$} & \multirow{3}{*}{$\begin{array}{c}\text { Exp. } \\
\text { determined } \\
\text { phases }\end{array}$} & \multicolumn{6}{|c|}{ Compositions of phases (at.\%) } \\
\hline & & & & \multicolumn{2}{|l|}{$\mathrm{Ag}$} & \multicolumn{2}{|l|}{$\mathrm{Bi}$} & \multicolumn{2}{|l|}{$\mathrm{Ga}$} \\
\hline & & & & exp. & calc. & exp. & calc. & exp. & calc. \\
\hline \multirow{3}{*}{1.} & 19. & $\mathrm{~L}^{\prime}$ & $\mathrm{L}^{\prime \prime}$ & $4.01 \pm 0.1$ & 2.81 & $57.13 \pm 0.5$ & 59.1 & $6 \pm 0.3$ & $\overline{8.09}$ \\
\hline & & $\mathrm{L}^{\prime}$ & $\mathrm{L}^{\prime}$ & & 6.6 & 32. & 31.5 & $06 \pm 0.5$ & 61.9 \\
\hline & 50. & $\mathrm{Ag}_{3} \mathrm{Ga}_{2}$ & $\mathrm{Ag}_{3} \mathrm{G}$ & 0.3 & 60 & $1.46 \pm 0.3$ & - & $7 \pm 0.5$ & 40 \\
\hline \multirow{3}{*}{2.} & 60.4 & (Bi) & (Bi) & $76 \pm 0.4$ & - & $98.6 \pm 0.2$ & 100 & $4 \pm 0.1$ & - \\
\hline & 21.8 & (Ag) & $(\mathrm{Ag})$ & .3 & 86.03 & 0.83 & 0.38 & 0.2 & 13.59 \\
\hline & 17.7 & $\zeta^{\prime}$ & $\zeta^{\prime}$ & $75.06 \pm 0.6$ & 75.08 & $1.44 \pm 0.3$ & - & $23.5 \pm 0.2$ & 24.92 \\
\hline \multirow{3}{*}{3.} & 31.3 & $\mathrm{~L}^{\prime \prime}$ & $\mathrm{L}^{\prime \prime}$ & $1.53 \pm 0.4$ & 0.89 & $89.18 \pm 0.2$ & 89.66 & $9.29 \pm 0.2$ & 9.45 \\
\hline & $49.18 \mathrm{Bi}$ & $\zeta^{\prime}$ & $\zeta^{\prime}$ & 65.76 & 66.85 & $1.41 \pm 0.2$ & - & $32.83 \pm 0.4$ & 33.15 \\
\hline & $19.50 \mathrm{Ga}$ & (Di & . & 001 & 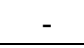 & & 100 & $137+0.4$ & - \\
\hline \multirow[b]{2}{*}{4.} & $30.54 \mathrm{Ag}$ & & & & 7.86 & 14.4 & 17.5 & $77.09 \pm 0.3$ & 74.64 \\
\hline & $\begin{array}{c}9.84 \mathrm{Bi} \\
59.62 \mathrm{Ga}\end{array}$ & $\mathrm{Ag}_{3} \mathrm{Ga}$ & $\mathrm{L}$ & 57.6 & 60 & 136 & - & $41+07$ & 40 \\
\hline \multirow[b]{2}{*}{5.} & & $(\mathrm{Bi}$ & $(\mathrm{Bi}$ & 0.3 & - & 98.2 & 100 & \pm 0.1 & - \\
\hline & $\begin{array}{l}23.18 \mathrm{~B} 1 \\
5.74 \mathrm{Ga}\end{array}$ & (Ag) & (Ag) & $90.58 \pm 0.5$ & 91.94 & $1.53 \pm 0.1$ & 0.42 & $7.89 \pm 0.3$ & 7.64 \\
\hline \multirow{3}{*}{6.} & $19.25 \mathrm{Ag}$ & $\mathrm{L}^{\prime \prime}$ & $\mathrm{L}^{\prime \prime}$ & $65 \pm 0.3$ & 0.89 & $89.5 \pm 0.7$ & 89.66 & $8.85 \pm 0.3$ & 9.45 \\
\hline & $68.42 \mathrm{Bi}$ & $\zeta^{\prime}$ & $\zeta^{\prime}$ & $65.91 \pm 0.6$ & 66.85 & $1.11 \pm 0.3$ & - & $32.98 \pm 0.3$ & 33.15 \\
\hline & $12.33 \mathrm{Ga}$ & (Bi) & (Bi) & $0.91 \pm 0.3$ & - & $98.18 \pm 0.3$ & 100 & $0.91 \pm 0.2$ & - \\
\hline
\end{tabular}


According to the results given in Table 2 the existence of the all predicted phases was confirmed. Phase $\mathrm{Ag}_{3} \mathrm{Ga}_{2}$ in the samples 1 and 4; has a solubility of $\mathrm{Bi}$ of around $1.4 \%$; the same value is detected for $\zeta^{\prime}-\mathrm{Ag}_{2} \mathrm{Ga}$ phase. Also; it can be seen that $\mathrm{Bi}$ phases have a very small solubility of $\mathrm{Ag}$ and Ga. Phase $\mathrm{L}^{\prime}$ is rich in Ga and $\mathrm{L}^{\prime \prime}$ is rich in $\mathrm{Bi}$ and this difference was predicted in binary Bi-Ga system. Fig. 2; presents microstructures of the six studied alloy samples. Phases identified using energy dispersive spectrometry (EDS) analysis are marked on the presented microstructures.
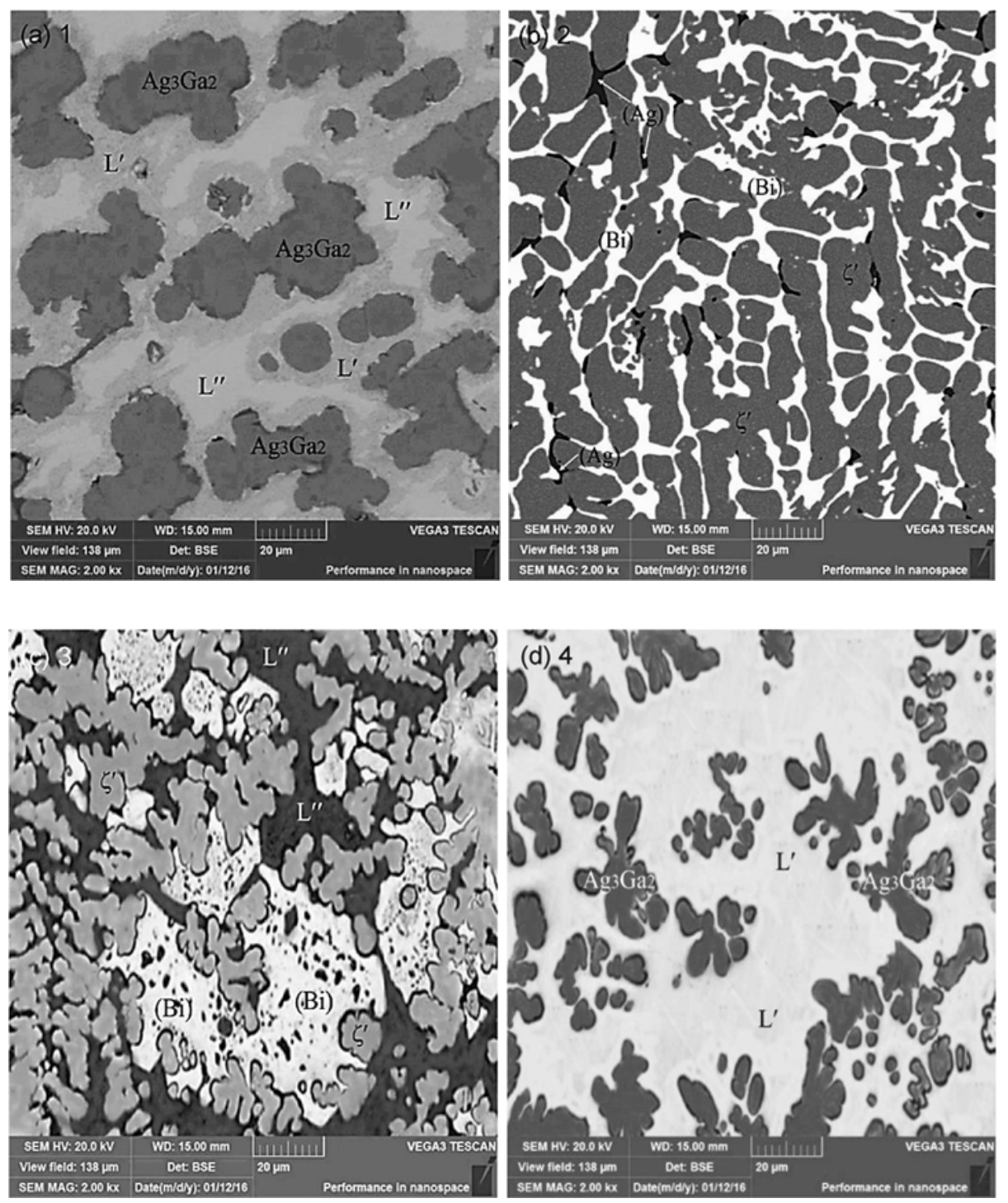

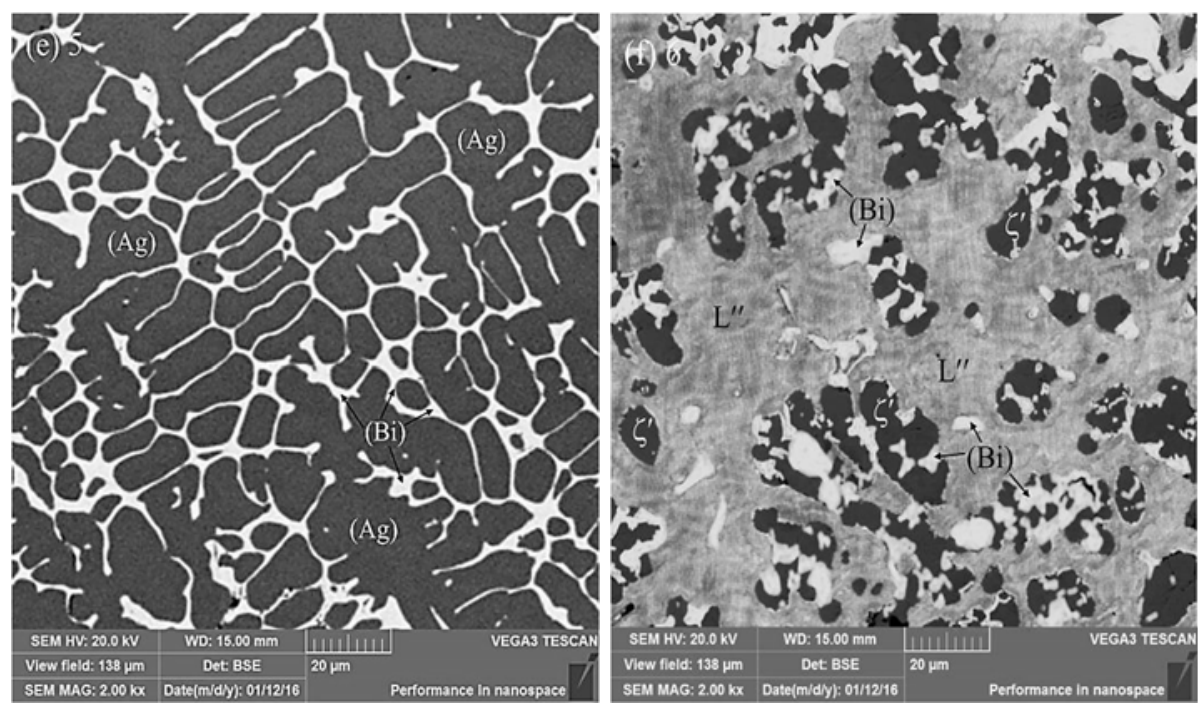

Fig. 2. Microstructures of the alloys analyzed by the SEM-EDS technique.

Additional seven samples from the isothermal section at $250{ }^{\circ} \mathrm{C}$ were investigated with XRD technique. Compositions of these samples are marked on Fig. 1 with Roman numerals from I to VII. The samples I and IV belong to the same threephase region $\mathrm{L}^{\prime \prime}+\zeta^{\prime}+(\mathrm{Bi})$; the samples III and $\mathrm{V}$ are from two-phase region $(\mathrm{Ag})+(\mathrm{Bi})$; the sample II is from two-phase region $\zeta^{\prime}+(\mathrm{Bi})$; sample VI is from $\mathrm{L}^{\prime \prime}+\zeta^{\prime}+\mathrm{Ag}_{3} \mathrm{Ga}_{2}$ region and the sample VII is from two-phase region $\mathrm{L}^{\prime \prime}+\mathrm{Ag}_{3} \mathrm{Ga}_{2}$. The same alloy samples were further analyzed by XRD technique. The obtained results of XRD analysis are shown in Table 3.

Table 3. Comparative presentation of the calculated and the experimentally obtained $X R D$ data for the studied alloy samples at $250^{\circ} \mathrm{C}$.

\begin{tabular}{|c|c|c|c|c|c|c|c|c|c|}
\hline \multirow{3}{*}{ S. } & \multirow{3}{*}{$\begin{array}{c}\text { Overall } \\
\text { composition } \\
\text { (at. \%) }\end{array}$} & \multicolumn{2}{|c|}{ Phase } & \multicolumn{6}{|c|}{ Lattice parameters $(\AA)$} \\
\hline & & \multirow{2}{*}{ Pred. } & \multirow{2}{*}{ Ident. } & \multicolumn{2}{|c|}{$a$} & \multicolumn{2}{|c|}{$b$} & \multicolumn{2}{|c|}{$c$} \\
\hline & & & & Exp. & Ref. & Exp. & Ref. & Exp. & Ref. \\
\hline \multirow{3}{*}{1} & $19.32 \mathrm{Ag}$ & $\mathrm{L}^{\prime \prime}$ & $\mathrm{L}^{\prime \prime}$ & & & & & & \\
\hline & $30.48 \mathrm{Bi}$ & $\mathrm{L}^{\prime}$ & $\mathrm{L}^{\prime}$ & & & & & & \\
\hline & $50.20 \mathrm{Ga}$ & $\mathrm{Ag}_{3} \mathrm{Ga}_{2}$ & $\mathrm{Ag}_{3} \mathrm{Ga}_{2}$ & $6.7245(2)$ & $6.7271[1]$ & $3.8753(3)$ & $3.8764[1]$ & $3.1808(5)$ & $3.1802[1]$ \\
\hline \multirow{3}{*}{2.} & $60.41 \mathrm{Ag}$ & (Bi) & (Bi) & $4.5694(3)$ & $4.5462[16]$ & & & $11.8576(1)$ & $11.8591[16]$ \\
\hline & $21.87 \mathrm{Bi}$ & $\zeta^{\prime}$ & $\zeta^{\prime}$ & $7.7482(2)$ & $7.7460[17]$ & & & $2.8698(6)$ & $2.8704[17]$ \\
\hline & $17.72 \mathrm{Ga}$ & $(\mathrm{Ag})$ & $(\mathrm{Ag})$ & $4.0889(4)$ & $4.094[18]$ & & & & \\
\hline \multirow{3}{*}{3.} & $31.32 \mathrm{Ag}$ & $\mathrm{L}^{\prime \prime}$ & $\mathrm{L}^{\prime \prime}$ & & & & & & \\
\hline & $49.18 \mathrm{Bi}$ & (Bi) & (Bi) & $4.5567(1)$ & $4.5462[16]$ & & & $11.8451(3)$ & $11.8591[16]$ \\
\hline & $19.50 \mathrm{Ga}$ & $\zeta^{\prime}$ & $\zeta^{\prime}$ & $7.7457(2)$ & $7.7460[17]$ & & & $2.8693(6)$ & $2.8704[17]$ \\
\hline \multirow{2}{*}{4.} & $30.54 \mathrm{Ag}$ & $\mathrm{L}^{\prime}$ & $\mathrm{L}^{\prime}$ & & & & & & \\
\hline & $\begin{array}{c}9.84 \mathrm{Bi} \\
59.62 \mathrm{Ga}\end{array}$ & $\mathrm{Ag}_{3} \mathrm{Ga}_{2}$ & $\mathrm{Ag}_{3} \mathrm{Ga}_{2}$ & $6.7287(5)$ & $6.7271[1]$ & $3.8765(2)$ & $3.8764[1]$ & $3.1843(5)$ & $3.1802[1]$ \\
\hline \multirow{2}{*}{5.} & $71.08 \mathrm{Ag}$ & (Bi) & (Bi) & $4.5465(3)$ & $4.5462[16]$ & & & $11.8576(7)$ & $11.8591[16]$ \\
\hline & $\begin{array}{l}23.18 \mathrm{Bl} \\
5.74 \mathrm{Ga}\end{array}$ & $(\mathrm{Ag})$ & $(\mathrm{Ag})$ & $4.0872(6)$ & $4.094[18]$ & & & & \\
\hline
\end{tabular}




\begin{tabular}{|c|c|c|c|c|c|c|c|c|}
\hline 6. & $\begin{array}{l}19.25 \mathrm{Ag} \\
68.42 \mathrm{Bi} \\
12.33 \mathrm{Ga}\end{array}$ & $\begin{array}{c}\mathrm{L}^{\prime \prime} \\
(\mathrm{Bi}) \\
\zeta^{\prime}\end{array}$ & $\begin{array}{c}\mathrm{L}^{\prime \prime} \\
(\mathrm{Bi}) \\
\zeta^{\prime}\end{array}$ & $\begin{array}{l}4.5498(2) \\
7.7445(6)\end{array}$ & $\begin{array}{l}4.5462[16] \\
7.7460[17]\end{array}$ & & $\begin{array}{c}11.8602(3) \\
2.8756(3)\end{array}$ & $\begin{array}{c}- \\
11.8591[16] \\
2.8704[17]\end{array}$ \\
\hline I & $\begin{array}{l}3 \mathrm{Ag} \\
93 \mathrm{Bi} \\
4 \mathrm{Ga}\end{array}$ & $\begin{array}{c}\mathrm{L}^{\prime \prime} \\
(\mathrm{Bi}) \\
\zeta^{\prime}\end{array}$ & $\begin{array}{c}\mathrm{L}^{\prime \prime} \\
(\mathrm{Bi}) \\
\zeta^{\prime}\end{array}$ & $\begin{array}{l}4.5476(8) \\
7.7487(8)\end{array}$ & $\begin{array}{l}4.5462[16] \\
7.7460[17]\end{array}$ & & $\begin{array}{c}11.8577(5) \\
2.8698(1)\end{array}$ & $\begin{array}{c}- \\
11.8591[16] \\
2.8704[17]\end{array}$ \\
\hline II & $\begin{array}{l}60 \mathrm{Ag} \\
18 \mathrm{Bi} \\
22 \mathrm{Ga}\end{array}$ & $\begin{array}{c}(\mathrm{Bi}) \\
\zeta^{\prime}\end{array}$ & $\begin{array}{c}(\mathrm{Bi}) \\
\zeta^{\prime}\end{array}$ & $\begin{array}{l}4.5561(1) \\
7.7458(3)\end{array}$ & $\begin{array}{l}4.5462[16] \\
7.7460[17]\end{array}$ & & $\begin{array}{l}11.8611(1) \\
2.8732(3)\end{array}$ & $\begin{array}{c}11.8591[16] \\
2.8704[17]\end{array}$ \\
\hline III & $\begin{array}{c}40 \mathrm{Ag} \\
56 \mathrm{Bi} \\
4 \mathrm{Ga}\end{array}$ & $\begin{array}{l}(\mathrm{Bi}) \\
(\mathrm{Ag})\end{array}$ & $\begin{array}{l}(\mathrm{Bi}) \\
(\mathrm{Ag})\end{array}$ & $\begin{array}{l}4.5531(2) \\
4.1003(1)\end{array}$ & $\begin{array}{c}4.5462[16] \\
4.094[18]\end{array}$ & & $11.8606(5)$ & $11.8591[16]$ \\
\hline IV & $\begin{array}{l}40 \mathrm{Ag} \\
42 \mathrm{Bi} \\
18 \mathrm{Ga}\end{array}$ & $\begin{array}{c}\mathrm{L}^{\prime \prime} \\
(\mathrm{Bi}) \\
\zeta^{\prime}\end{array}$ & $\begin{array}{c}\mathrm{L}^{\prime \prime} \\
(\mathrm{Bi}) \\
\zeta^{\prime}\end{array}$ & $\begin{array}{l}4.5676(1) \\
7.7458(2)\end{array}$ & $\begin{array}{l}4.5462[16] \\
7.7460[17]\end{array}$ & & $\begin{array}{c}11.8566(7) \\
2.8713(5)\end{array}$ & $\begin{array}{c}11.8591[16] \\
2.8704[17]\end{array}$ \\
\hline $\mathrm{V}$ & $\begin{array}{l}80 \mathrm{Ag} \\
13 \mathrm{Bi} \\
7 \mathrm{Ga}\end{array}$ & $\begin{array}{l}(\mathrm{Bi}) \\
(\mathrm{Ag})\end{array}$ & $\begin{array}{l}\text { (Bi) } \\
\text { (Ag) }\end{array}$ & $\begin{array}{l}4.5576(1) \\
4.1001(7)\end{array}$ & $\begin{array}{c}4.5462[16] \\
4.094[18]\end{array}$ & & $11.8603(7)$ & $11.8591[16]$ \\
\hline VI & $\begin{array}{l}40 \mathrm{Ag} \\
33 \mathrm{Bi} \\
27 \mathrm{Ga}\end{array}$ & $\begin{array}{c}\mathrm{L}^{\prime \prime} \\
\zeta^{\prime} \\
\mathrm{Ag}_{3} \mathrm{Ga}_{2} \\
\end{array}$ & $\begin{array}{c}\mathrm{L}^{\prime \prime} \\
\zeta^{\prime} \\
\mathrm{Ag}_{3} \mathrm{Ga}_{2} \\
\end{array}$ & $\begin{array}{l}7.7454(3) \\
6.7251(3)\end{array}$ & $\begin{array}{c}7.7460[17] \\
6.7271[1]\end{array}$ & $3.8763(5) 3.8764[1]$ & $\begin{array}{l}2.8712(1) \\
3.1813(1)\end{array}$ & $\begin{array}{c}2.8704[17] \\
3.1802[1] \\
\end{array}$ \\
\hline VII & $\begin{array}{l}20 \mathrm{Ag} \\
53 \mathrm{Bi} \\
27 \mathrm{Ga}\end{array}$ & $\begin{array}{c}\mathrm{L}^{\prime \prime} \\
\mathrm{Ag}_{3} \mathrm{Ga}_{2}\end{array}$ & $\begin{array}{c}\mathrm{L}^{\prime \prime} \\
\mathrm{Ag}_{3} \mathrm{Ga}_{2}\end{array}$ & $6.7251(3)$ & $6.7271[1]$ & $3.8763(5) \quad 3.8764[1]$ & $3.1813(1)$ & $3.1802[1]$ \\
\hline
\end{tabular}

Obtained values of lattice parameters for $\mathrm{Ag}_{3} \mathrm{Ga}_{2} ;(\mathrm{Bi}) ; \zeta^{\prime}$ and $(\mathrm{Ag})$ phases are in close agreement with literature data [1, $16-18]$. The experimentally identified phases by SEM-EDS are same as detected by XRD analysis. XRD pattern of sample I with identified phases is shown on Fig. 3 as an illustration.

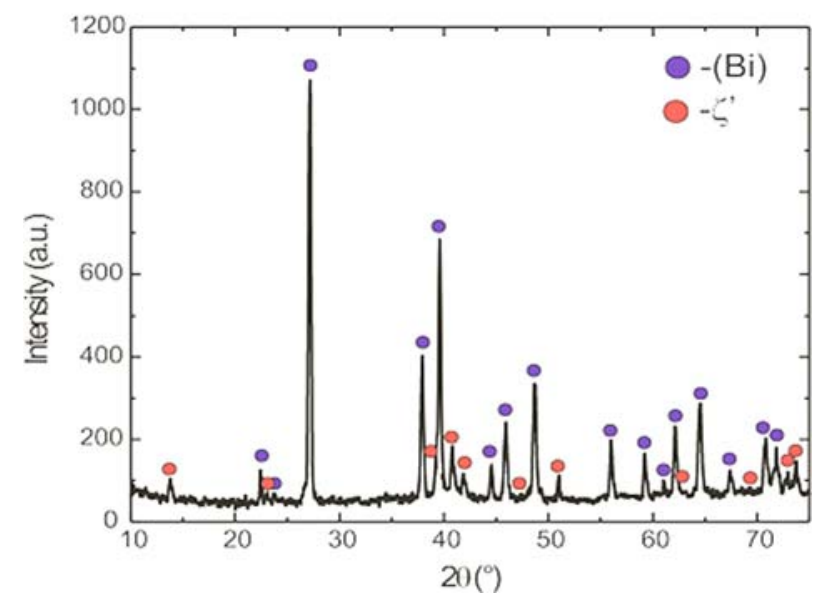

Fig. 3. XRD pattern for the sample alloy I.

Vickers microhardness was investigated using the same alloys samples investigated by XRD. Compositions of these thirteen alloys are marked on Fig. 1. The load of $0.245 \mathrm{~N}$ was applied and time of loading was $20 \mathrm{~s}$. The values of Vickers microhardness are given in Table 4. Vickers microhardness for each phase was 
determined by five measurements and in Table are given a mean value for investigated phases.

Table 4. Measured Vickers microhardness of the phases in ternary Ag-Ga-Bi system.

\begin{tabular}{|c|c|c|c|c|c|c|c|c|c|c|c|c|c|}
\hline \multirow{3}{*}{$\begin{array}{l}\text { Determined } \\
\text { phases }\end{array}$} & \multicolumn{13}{|c|}{ Sample } \\
\hline & 1 & 2 & 3 & 4 & 5 & 6 & I & II & III & IV & V & VI & VII \\
\hline & \multicolumn{13}{|c|}{ Mean value $\left(\mathrm{MN} \mathrm{x} \mathrm{m}^{-2}\right)$} \\
\hline $\mathrm{Ag}_{3} \mathrm{Ga}_{2}$ & 130.5 & & & 131.8 & & & & & & & & 132.1 & 131.5 \\
\hline (Bi) & & 95.5 & 96.2 & & 93.5 & 95.4 & 90.6 & 91.5 & 93.8 & 90.5 & 94.6 & & \\
\hline$\zeta^{\prime}$ & & 146.4 & 140.3 & & & 141.9 & 143.4 & 144.6 & & 143.3 & & 145.2 & \\
\hline$(\mathrm{Ag})$ & & 55.7 & & & 54.7 & & & & 53.7 & & 56.2 & & \\
\hline
\end{tabular}

From all investigated phases; phase rich in silver; solid solution (Ag) has the lowest value of hardness in range 53.7 to $56.2 \mathrm{MN} \mathrm{m}^{-2}$. Mean value of hardness for solid solution (Bi) phase is $93.51 \mathrm{MN} \mathrm{m}{ }^{-2}$. Other two phases $\zeta^{\prime}-\mathrm{Ag}_{2} \mathrm{Ga}$ and $\mathrm{Ag}_{3} \mathrm{Ga}_{2}$ have almost the same hardness. Mean value of hardness for $\zeta^{\prime}-\mathrm{Ag}_{2} \mathrm{Ga}$ is $143.59 \mathrm{MN} \mathrm{m}^{-2}$ and for $\mathrm{Ag}_{3} \mathrm{Ga}_{2}$ is slightly lower $131.48 \mathrm{MN} \mathrm{m}^{-2}$.

The hardness of the ternary Ag-Bi-Ga alloys was determined using Brinell test method. As aforementioned; the alloy samples were selected from the three vertical sections: $\mathrm{Bi}-\mathrm{AgGa}$; $\mathrm{Ag}-\mathrm{BiGa}$; and $\mathrm{Ga}-\mathrm{AgBi}$. Summary of the obtained experimental results is given in Table 5; together with literature values of hardness for pure elements [19].

Table 5. Brinell hardness of the alloys of the ternary Ag-Bi-Ga system.

\begin{tabular}{ccccccc}
\hline \multicolumn{3}{c}{ Mole fraction of components } & \multicolumn{3}{c}{$\begin{array}{c}\text { Hardness of Brinell } \mathrm{HB}\left(\mathrm{MN} \mathrm{x} \mathrm{m}^{-2}\right) \\
\text { measurement }\end{array}$} & Mean value \\
\cline { 1 - 5 } $\mathrm{x}(\mathrm{Bi})$ & $\mathrm{x}(\mathrm{Ag})$ & $\mathrm{x}(\mathrm{Ga})$ & 1 & 2 & 3 & \\
\hline 0 & 0.5 & 0.5 & 45.1 & 41.0 & 43.05 & 43.05 \\
0.2 & 0.4 & 0.4 & 39.1 & 47.4 & 46.7 & 44.4 \\
0.4 & 0.3 & 0.3 & 53.68 & 51.22 & 52.12 & 52.34 \\
0.6 & 0.2 & 0.2 & 72.1 & 74.1 & $75 ; 7$ & 73.6 \\
0.8 & 0.1 & 0.1 & 82.07 & 80.23 & 82.26 & 81.52 \\
1 & 0 & 0 & & & & 94.2 \\
0.5 & 0 & 0.5 & 27.3 & 30.5 & 28.9 & 28.9 \\
0.4 & 0.2 & 0.4 & 36.46 & 35.82 & 37.64 & 36.64 \\
0.3 & 0.4 & 0.3 & 76.6 & 78.1 & 77.5 & 77.4 \\
0.2 & 0.6 & 0.2 & 52.1 & 41.5 & 48.7 & 47.4 \\
0.1 & 0.8 & 0.1 & 26.06 & 27.96 & 27.82 & 27.28 \\
0 & 1 & 0 & & & & 24.5 \\
0.5 & 0.5 & 0 & 38.2 & 38.0 & 38.1 & 38.1 \\
0.4 & 0.4 & 0.2 & 35.89 & 33.96 & 35.87 & 35.24 \\
0.3 & 0.3 & 0.4 & 45.8 & 49.0 & 47.8 & 47.5 \\
0.2 & 0.2 & 0.6 & 56.2 & 56.7 & 53.5 & 55.4 \\
0.1 & 0.1 & 0.8 & 54.21 & 54.34 & 58.49 & 55.68 \\
0 & 0 & 1 & & & & 60 \\
\hline
\end{tabular}


A graphical presentation of the relationship between the Brinell hardness of the studied alloys from the three vertical sections and the alloy composition is given in Fig. 4.
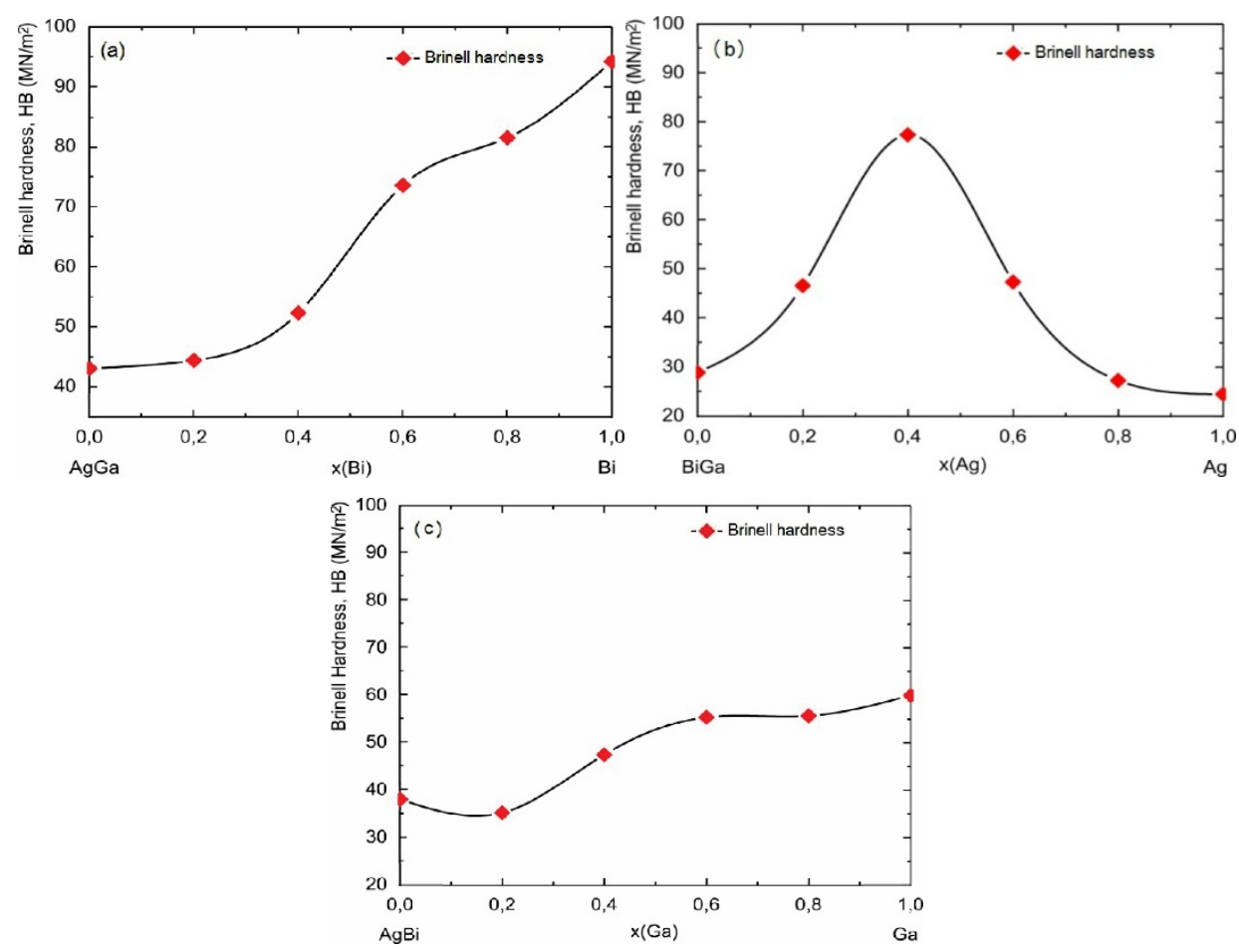

Fig. 4. Brinell hardness for investigated samples from isothermal sections at $250^{\circ} \mathrm{C}$.

Experimentally determined values of hardness given in Table 5; and an appropriate mathematical model were used to calculate values of hardness along the isothermal section at $250{ }^{\circ} \mathrm{C}$ of the ternary Ag-Bi-Ga system.

In order to define a mathematical model for the dependence of Brinell hardness vs composition for Ag-Bi-Ga alloys the Design Expert v.9.0.3.1 software package is used. Out of a possible canonical or Scheffe model [20 - 22] that meet the requirements of adequacy a Special Cubic Mixture Model is recommended:

$$
\hat{y}=\sum_{i=1}^{q} \beta_{i} x_{i}+\sum_{i<j}^{q-1} \sum_{j}^{q} \beta_{i j} x_{i} x_{j}+\sum_{i<j}^{q-2} \sum_{j<k}^{q-1} \sum_{k}^{q} \beta_{i j k} x_{i} x_{j} x_{k}
$$

The Analysis of variance (ANOVA) confirms the adequacy of the Model. The Fvalue of the Model is 10.855 and it implies that the model is significant. There is only a $0.01 \%$ chance that a "Model F-Value" this large could occur due to noise. The final equation of the predictive model in terms of actual components is:

$\mathrm{HB}=106.3431 \cdot \mathrm{Bi}+18.52489 \cdot \mathrm{Ag}+44.38481 \cdot \mathrm{Ga}-93.6128 \cdot \mathrm{Bi} \cdot \mathrm{Ag}-170.947 \cdot \mathrm{Bi} \cdot \mathrm{Ga}+$ 
Iso-lines contour plot for Brinell hardness of alloys defined by Equation 2 is shown in Fig. 5.

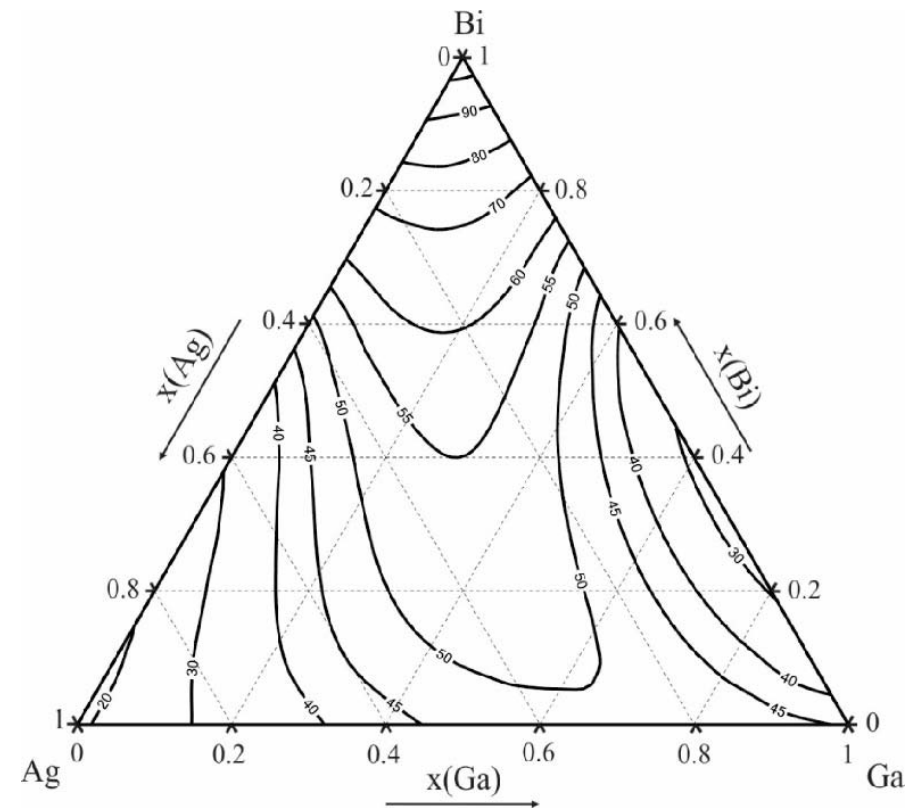

Fig. 5: Iso-lines of Brinell Hardness of the ternary Ag-Bi-Ga system at $250^{\circ} \mathrm{C}$.

Table 6 presents the obtained experimental results of electrical conductivity measurements and literature values of electrical conductivity for pure $\mathrm{Ag}, \mathrm{Bi}$, and $\mathrm{Ga}$ [23].

Table 6. Electrical conductivity of the alloys from isothermal sections at $250{ }^{\circ} \mathrm{C}$.

\begin{tabular}{cccccccc}
\hline \multicolumn{3}{c}{$\begin{array}{c}\text { Mole fraction of } \\
\text { components }\end{array}$} & \multicolumn{5}{c}{$\begin{array}{c}\text { Electrical conductivity }\left(\mathrm{MS} \mathrm{m}^{-1}\right) \\
\text { Values for different measurement }\end{array}$} \\
\cline { 1 - 6 } $\mathrm{x}(\mathrm{Bi})$ & $\mathrm{x}(\mathrm{Ag})$ & $\mathrm{x}(\mathrm{Ga})$ & 1 & 2 & 3 & 4 & \\
\hline 0 & 0.5 & 0.5 & 1.1637 & 1.2428 & 1.3058 & 1.2325 & 1.2362 \\
0.2 & 0.4 & 0.4 & 1.31 & 1.315 & 1.326 & 1.306 & 1.3143 \\
0.4 & 0.3 & 0.3 & 1.915 & 1.863 & 1.786 & 1.96 & 1.8810 \\
0.6 & 0.2 & 0.2 & 0.5518 & 0.5696 & 0.5634 & 0.6054 & 0.5726 \\
0.8 & 0.1 & 0.1 & 0.5154 & 0.5065 & 0.5084 & 0.4955 & 0.5065 \\
1 & 0 & 0 & & & & & 0.77 \\
0.5 & 0 & 0.5 & 1.3124 & 1.3482 & 1.3124 & 1.3326 & 1.3264 \\
0.4 & 0.2 & 0.4 & 1.461 & 1.462 & 1.518 & 1.543 & 1.4960 \\
0.3 & 0.4 & 0.3 & 1.529 & 1.662 & 1.727 & 1.629 & 1.6368
\end{tabular}




\begin{tabular}{cccccccc}
0.2 & 0.6 & 0.2 & 2.103 & 2.12 & 2.109 & 2.093 & 2.1063 \\
0.1 & 0.8 & 0.1 & 6.4324 & 6.4528 & 6.4486 & 6.4354 & 6.4423 \\
0 & 1 & 0 & & & & & 62 \\
0.5 & 0.5 & 0 & 1.8826 & 1.9342 & 1.9677 & 1.9227 & 1.9268 \\
0.4 & 0.4 & 0.2 & 1.52 & 1.481 & 1.508 & 1.507 & 1.5040 \\
0.3 & 0.3 & 0.4 & 1.32 & 1.371 & 1.347 & 1.348 & 1.3465 \\
0.2 & 0.2 & 0.6 & 1.146 & 1.126 & 1.207 & 1.135 & 1.1535 \\
0.1 & 0.1 & 0.8 & 1.773 & 1.472 & 1.658 & 1.734 & 1.6593 \\
0 & 0 & 1 & & & & & 7.1 \\
\hline
\end{tabular}

Graphical presentation of the correlation between electrical conductivity and mole fraction of components for the all investigated samples is shown in Fig. 6.
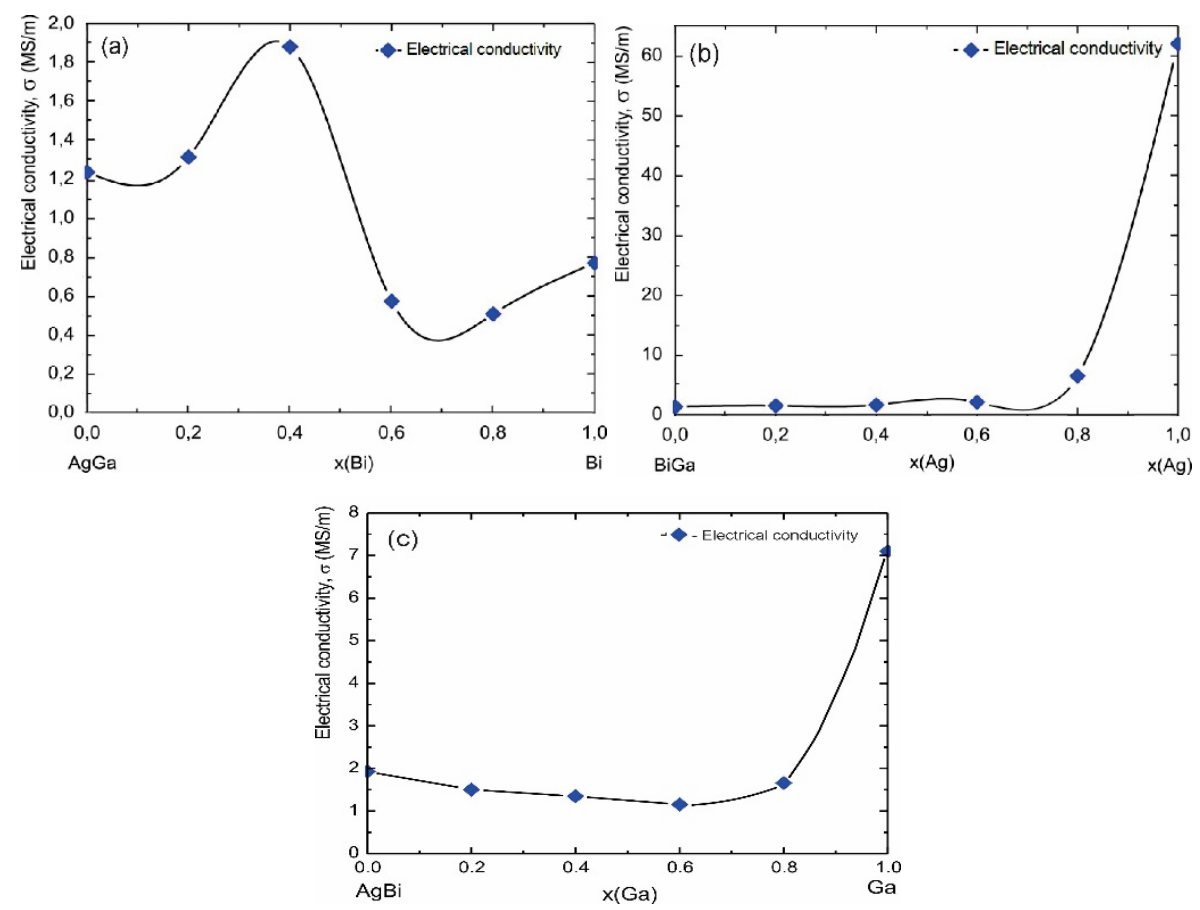

Fig. 6. Electrical conductivity for investigated samples from isothermal sections at $250^{\circ} \mathrm{C}$.

Calculation of electrical conductivity for the alloys from the Ag-Bi-Ga ternary system was carried out in the same manner as the aforementioned Brinell hardness calculation.

Model summary statistics are suggested Special Cubic Mixture Model. The Fvalue of the Model is 8.89 in the Analysis of variance (ANOVA) and it implies that the 
model is significant. The final equation of the predictive model in Terms of Actual Components is:

$$
\sigma=4.818142 \cdot B i+27.64718 \cdot A g+8.983279 \cdot G a-72.3709 \cdot B i \cdot A g-18.314 \cdot B i \cdot G a-
$$

84.8721 $\cdot \mathrm{Ag} \cdot \mathrm{Ga}+173.949 \cdot \mathrm{Bi} \cdot \mathrm{Ag} \cdot \mathrm{Ga}$

Iso-lines contour plot of Electric Conductivity defined by equation 3 is shown in Fig. 7.

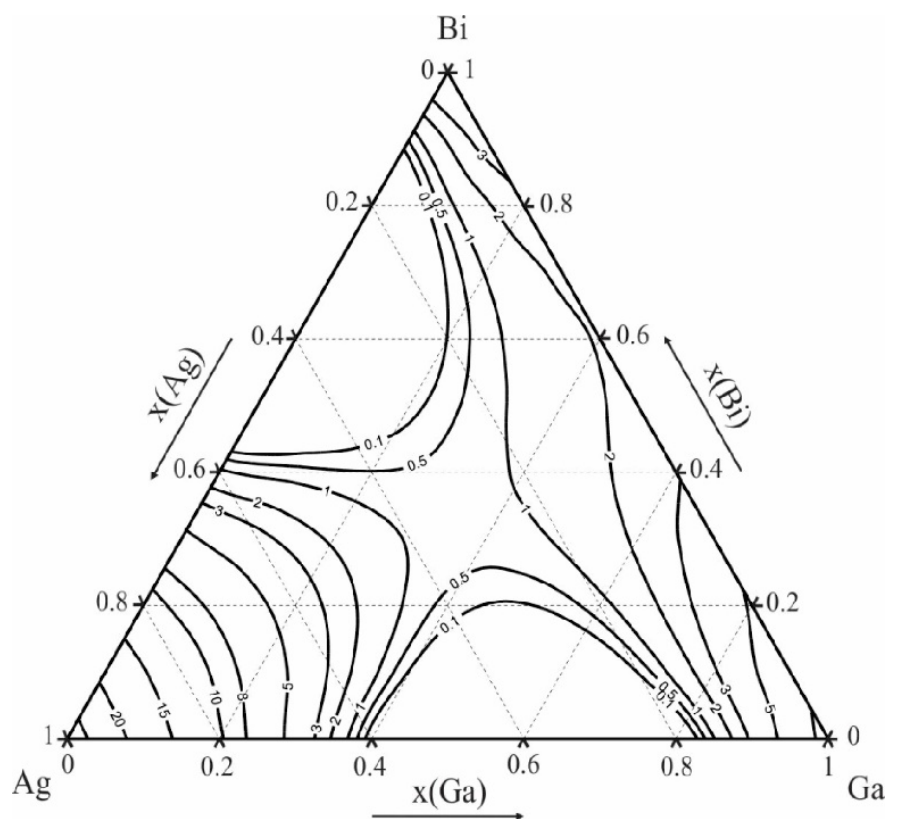

Fig. 7. Iso-lines of electrical conductivity of ternary Ag-Bi-Ga system at $250^{\circ} \mathrm{C}$.

Based on experimental results and used a mathematical model for calculation of electrical conductivity for all alloys in ternary Ag-Bi-Ga system at $250^{\circ} \mathrm{C}$ it is clear that all Ag-rich alloys will have better electrical conductivity than Bi-rich or Ga-rich alloys.

Similar studies on other ternary systems were published by the same group of authors in past $[24,25]$ because temperature-related changes of mechanical and electrical properties of alloys are very important parameters that determine their possible future applications.

\section{Conclusion}

In this study; an isothermal section of the ternary Ag-Bi-Ga system at $250{ }^{\circ} \mathrm{C}$ was experimentally investigated and thermodynamically extrapolated using optimized thermodynamic unary and binary data taken from literature. Microstructures and phase compositions of the alloys annealed at $250{ }^{\circ} \mathrm{C}$ were studied using SEM-EDS and XRD analysis. The experimentally obtained results were found to be in a close agreement with the predicted phase diagram at $250^{\circ} \mathrm{C}$. Vickers microhardness measurements were 
carried out for the phases present in the microstructure of the eleven selected alloy samples. Values of Brinell hardness and electrical conductivity were experimentally determined for the selected alloys from the $\mathrm{Bi}-\mathrm{AgGa}$; $\mathrm{Ag}-\mathrm{BiGa}$; and $\mathrm{Ga}-\mathrm{AgBi}$ vertical sections of the ternary Ag-Bi-Ga system. Based on the experimentally obtained results iso-lines of Brinell hardness and electrical conductivity for the entire compositional range of the ternary system were calculated.

\section{Acknowledgements}

This work was supported by the Ministry of Education; Science and Technological Development of the Republic of Serbia; under Project No. ON172037 and 2011 Union-Innovative center of the Central South University of China. The financial support by the project CEITEC 2020 (LQ1601) from the Ministry of Education; Youth and Sports of the Czech Republic under the National Sustainability Programme II is gratefully acknowledged. Calculations were performed by Pandat 8.1 software.

\section{References}

[1] D. Minic, M. Premovic, D. Manasijevic, V. Cosovic, D. Zivkovic, A. Markovic: J Alloys Compd, 646 (2015) 461-471.

[2] The periodic table of the elements, http://www.webelements.com/thermochemistry.html, accessed 23.01.2016.

[3] M. Premović, D. Minić, D. Manasijević, Vladan Ćosović, D. Živković, I. Dervišević, N. Talijan: Acta Metall. Sin. (Engl. Lett.), 27 (2014) 47-54.

[4] D. Minić, M. Premović, D. Manasijević, D. Živković, D. Čikara: Mater Test, 56 (2014) 667-674.

[5] D. Minić, M. Premović, D. Živković, D. Manasijević, M. Dimić, Z. Petrović S. Marković: Rev Metal, 51 (2015) e042.

[6] G. Garzel, M. Kopyto, L.A.Zabdyr: J Min Metall, Sect B, 50 (2014) 145-148.

[7] I. Ohnuma, R. Kainuma, K. Ishida: J Min Metall, Sect B, 48 (2012) 413-418.

[8] D. Li, S. Delsantea, W. Gong, G. Borzone: Thermochim Acta, 523 (2011) 51-62.

[9] Y. Zhang, J. B. Li, J. K. Liang, Q.L. Liu, Y. G. Xiao, Q. Zhang, G. H. Rao, C. R. Li: CALPHAD: Comput Coupling Phase Diagrams Thermochem, 30 (2006) 316322.

[10] W. Gierlotka, D. Jendrzejczyk-Handzlik: J Alloys Compd, 509 (2011) 38-42.

[11]E. Zoro, C. Servant, B. Legendre: Computer Coupling of Phase Diagrams and Thermochemistry 31 (2007) 89-94.

[12]C. Girard: Thesis, University of Provence, Marseille (1985).

[13]W. Cao, S. L. Chen, F. Zhang, K. Wu, Y. Yang, Y. A. Chang, R. Schmid-Fetzer, W.A. Oates: CALPHAD: Comput Coupling Phase Diagrams Thermochem, 33 (2009) 328-342.

[14]A. Dinsdale, A. Watson, A. Kroupa, J. Vrestal, A. Zemanova, J. Vizdal: COST Action 531-Atlas of Phase Diagrams for Lead-Free Soldering, COST Office, Brussels (2008).

[15] A. Dinsdale, A. Kroupa, A. Watson, J. Vrestal, A. Zemanova, P. Broz, COST Action 602-Handbook of High-temperature Lead-free Solders: Atlas of Phase Diagrams, COST Office, Brussels (2012). 
[16]R. A. Robinson, A. Purwanto, M. Koghi, P. C. Canfield, T. Kamiyama, T. Ishigaki, J. W. Lynn, R. Erwin, E. Peterson, R. Movshovich: Phys Rev B, 50 (1994) 95959598.

[17]A. E. Gunnaes, A. Olsen, P.T. Zagierski, B. Klewe, O.B. Karlsen, A. Aasen: Z Kristallogr, 213 (1998) 639-644.

[18]X. S. Yan, P. Lin, X. Qi, L. Yang: Int J Mater Res, 102 (2011) 381-388.

[19] Hardness - Brinell: periodicity, http://www.webelements.com/periodicity/hardness_brinell/, accessed 24.01.2016.

[20] Cornell: Experiments with Mixtures, $3^{\text {rd }}$ Ed., Johñ Wiley\&Sons, Inc, New York (2002)

[21]Ž. Lazić, Design of Experiments in Chemical Engineering, Wilez-VCH Verlag GmbH\&Co.KGaA, Weiheim (2004).

[22] M. Kolarević, M. Vukićević, B. Radičević, M. Bjelić, V. Grković, A methodology for forming the regression model of ternary system, Faculty Of Mechanical Engineering, The Seventh Triennial International Conference Heavy Machinery HM 2011, Vrnjačka Banja (2011) 1-6.

[23] Electrical Conductivity of the elements, http://periodictable.com/Properties/A/ElectricalConductivity.an.html, accessed 24.01.2016.

[24] M. Premović, D. Minić, I. Manasijević, D. Živković: Mater Test, 57 (2015) 883888.

[25]D. Minić, M. Premović, M. Kolarević, V. Ćosović, D. Manasijević, D. Živković: J Mater Eng Perform, 22 (2013) 2343-2350.

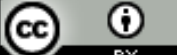

Creative Commons License

This work is licensed under a Creative Commons Attribution 4.0 International License. 